\title{
Bayesian prediction of future observations from inverse Weibull distribution based on type-II hybrid censored sample
}

\author{
Sanjay Kumar Singh, Umesh Singh, Vikas Kumar Sharma* \\ Department of Statistics and DST-CIMS, Banaras Hindu University, Varanasi, Pin-221005, India \\ ${ }^{*}$ Corresponding author E-mail: vikasstats@rediffmail.com
}

\begin{abstract}
In this paper, we have discussed the Bayesian procedure for the prediction of the future samples from inverse Weibull (IW) distribution under Type-II hybrid censoring scheme. Bayes estimators along with the corresponding highest posterior density (HPD) credible intervals have also been constructed for the parameters of IW distribution. The performance of the Bayes estimators of the model parameters has been compared with the maximum likelihood estimators through Monte Carlo Markov chain (MCMC) techniques. Finally, a real data set has been analysed to illustrate the discussed methodology.
\end{abstract}

Keywords: Hybrid censored sample, Bayes prediction, Predictive bounds, Gibbs Sampler and Metropolis-Hastings Algorithm.

\section{Introduction}

Prediction of the future samples based on current sample information (called informative sample) is very interesting topic in Statistics. The statistician, engineers, and other applied scientists have been using the prediction techniques for the various purposes. In Reliability analysis, life-tests are performed to observed the life of the experimental units put on test. In such a life test, some surviving units are removed or lost due to time and cost constraints or due to immediate needs of the units for other purposes. The data obtained from such a life-test are generally censored samples. In practice, the experimenter would like to know the failure times of the removed surviving units (censored units) based on observed data. This problem leads to the prediction of the future sample based on censored sample.

The conventional Type-I and Type-II censoring schemes have been most widely discussed in Reliability/Survival theory. Both Type-I and Type-II censoring scheme have their advantages and disadvantages. In Type-I censoring scheme, the test is terminated as soon as pre-fixed $\left(T_{0}\right)$ time has been reached while in Type-II censoring scheme, the test is stopped as soon as pre-fixed number $(R)$ items have failed. Thus, Type-I censoring scheme controls the duration of the test and efficiency of the test may be too low as the number of failure items is random where as Type-II censoring scheme controls the efficiency of the test and the termination time of the test is random since failure of the $R$ th item is uncertain.

[10] have introduced a more flexible censoring scheme, a mixture of Type-I and Type-II censoring schemes, named as hybrid censoring scheme. Hybrid censoring scheme is of two types namely (i) Type-I, and (ii) Type-II hybrid censoring schemes like as the conventional Type-I and Type-II censoring scheme. In Type-I hybrid censoring, the test is terminated at a time $T_{1}=\min \left(X_{R}, T_{0}\right)$, where $X_{R}$ represents the failure time of the $R$ th item and $T$ is the pre-fixed maximum allowable time of the test. In Type-II hybrid censoring, the test is terminated at a time $T_{2}=\max \left(X_{R}, T_{0}\right)$. It is clear that the test have at least $R$ failure items in Type-II hybrid censoring scheme where as in Type-I hybrid censoring scheme, the test can never be reached beyond the time $T_{0}$. Many authors have discussed the estimation procedures of the parameters of the various life-time models under Type-I hybrid, see for example $[21,16,24,12]$ and Type-II hybrid $[6,11,23,2]$ censoring schemes.

[19] and [7] have derived the density of the $r$ th future sample by using the concept of the Bayesian predictive 
posterior from Type-II censored sample follow the exponential distribution. Since then, Bayesian prediction problems for Type-I and Type-II censored data have been discussed by several authors, see the references cite in [1] but the limited attention has been paid for Bayesian prediction under hybrid censoring schemes. For exponential distribution under Type-I hybrid censoring scheme, [8] have discussed the two-sample Bayesian prediction of the future lifetimes and [9] have developed the classical prediction intervals for future failures. Recently, [1] and [26] considered a general form for the underlying distribution along with its general conjugate prior and have discussed the procedure for determining the one and two-sample Bayesian prediction intervals for future lifetimes based on a Type-II and Type-I hybrid censored sample respectively.

The inverse Weibull distribution has the following density function

$$
f(x)=\alpha \lambda x^{-\alpha-1} e^{-\lambda x^{-\alpha}}, x>0, \alpha, \lambda>0
$$

The cumulative distribution function (CDF) of (1) is given by

$$
F(x)=e^{-\lambda x^{-\alpha}} x>0, \alpha, \lambda>0
$$

The inverse Weibull distribution is found to be very appropriate over Weibull distribution when data indicates the non-monotone hazard rate. There are various real life examples where data don't shows the monotone hazard rate. For example, [18] have studied breast cancer data and observed that the mortality increases initially, reaches to a peak after some time and then declines slowly i.e., associated hazard rate is modified bathtub or particularly uni-modal. Such types of data can be modelled through IW distribution.

Since last decade, the inverse Weibull distribution is increasingly attracting the attention of the researchers. [4] have discussed the Two-sample Bayesian prediction for inverse Weibull distribution from complete sample of observations. [17] have derived the one and two sample predictive posteriors of the future order statistics from IW distribution under Type-II censoring scheme. Recently, [27] have discussed the classical as well as Bayesian estimation procedures for the estimation of the unknown parameters of inverse Weibull distribution under conventional Type-I and Type-II censoring schemes. In addition, [14, 15, 29, 22] can be referred to the readers for more detail on inverse Weibull distribution and related inferences. All references cited above discuss the estimation procedures for IW distribution under only complete sample of observations or Type-II censoring but not for more realistic and advanced censoring scheme such as hybrid censoring, progressive censoring and progressive hybrid censoring schemes etc. In this paper, we, therefore, have developed the Bayesian procedures for the estimation of the parameters and prediction of the future observations from IW distribution under Type-II hybrid censoring scheme.

In the lieu of above, the rest of the paper has been organized in the following sections. In section 2, the likelihood function has been derived for Type-II hybrid censored sample. The Bayesian estimation procedure for the estimation of the parameters of IW distribution has been developed in section 3. In section 4, a Monte Carlo simulation study has been carried out to compare the performance of the maximum likelihood and Bayes estimators of the model parameters. In section 5, one and two sample predictive posteriors of future samples have been derived. A real data set has been analysed in section 6 . Finally, conclusions have been given in section 7 .

\section{Likelihood based on Type-II hybrid censored sample}

Let $\mathrm{n}$ identical units follow the inverse Weibull distribution (1) are put on test and the test is terminated at a prespecified time (say, $T$ ) or when pre-determined number of failures (say, $R$ ) whichever occurs later i.e. $\max \left(X_{R: n}, T\right)$. Under such a type of experiment, the data can be obtained in the following ways:

Case I: $\left\{X_{1: n}, X_{2: n}, \ldots, X_{R: n}\right\}$ if $X_{R: n}>T, R<n$

Case II: $\left\{X_{1: n}, X_{2: n}, \ldots, X_{k: n}\right\}$ if $X_{k: n} \leq T<X_{k+1: n}, R \leq k \leq n$

It is clear that this sampling procedure provides at least $R$ sampling units for further analysis. The sample $\left\{X_{R+1: n}, X_{R+2: n}, \ldots, X_{n: n}\right\}$ and $\left\{X_{k+1: n}, X_{k+2: n}, \ldots, X_{n: n}\right\}$ are unobserved sampling units in case-I and case-II respectively. Then, the likelihood function under such Type-II hybrid censoring scheme can be defined as

$$
L=\frac{n !}{\left(n-n_{0}\right) !}(\alpha \lambda)^{n_{0}} \prod_{i=1}^{n_{0}} x_{i}^{-\alpha-1} e^{-\lambda \sum_{i=1}^{n_{0}} x_{i}^{-\alpha}}\left(1-e^{-\lambda t_{0}^{-\alpha}}\right)^{n-n_{0}}
$$

Where, $\quad n_{0}=\left\{\begin{array}{ll}R & \text { for case-I } \\ k & \text { for case-II }\end{array}, \quad t_{0}= \begin{cases}X_{R: n} & \text { for case-I } \\ T & \text { for case-II }\end{cases}\right.$ 
The MLEs $\hat{\alpha}$ and $\hat{\lambda}$ of $\alpha$ and $\lambda$ respectively, can be obtained as the pair of two real values for which likelihood function is to be maximised. Alternatively, for optimizing the log-likelihood function with respect to the involved parameters, one can use the inbuilt packages like $n \operatorname{lm}()$ and optim() of the R-software [25]. The log-likelihood function is given by

$$
\log L=\ln (n !)-\ln \left(\left(n-n_{0}\right) !\right)+n_{0} \ln (\alpha)+n_{0} \ln (\lambda)-(\alpha+1) \sum_{i=1}^{n_{0}} \ln \left(x_{i}\right)-\lambda \sum_{i=1}^{n_{0}} x_{i}^{-\alpha}+\left(n-n_{0}\right) \ln \left(1-e^{-\lambda t_{0}^{-\alpha}}\right)
$$

\section{Bayes estimation}

In this section, we have discussed the Bayesian estimation procedure for the parameters of IW distribution under Hybrid Type-II censoring scheme. In Bayesian set-up, we need to assume a prior distribution of the model parameters. Here, we assumed that the model parameters $\alpha$ and $\lambda$ follow the independent gamma prior density of the following forms:

$$
\begin{aligned}
& g(\alpha) \propto \alpha^{b-1} e^{-\alpha a} ; a, b, \alpha>0 \\
& g(\lambda) \propto \lambda^{d-1} e^{-\lambda c} ; c, d, \beta>0
\end{aligned}
$$

Where, $a, b, c$ and $d$ are the hyper-parameters. Then, the joint posterior PDF of $\alpha$ and $\beta$ is defined as

$$
\pi(\alpha, \lambda \underset{\sim}{x})=C^{\star} \alpha^{n_{0}+b-1} \lambda^{n_{0}+d-1} \prod_{i=1}^{n_{0}} x_{i}^{-\alpha-1} e^{-\alpha a-\lambda\left(\sum_{i=1}^{n_{0}} x_{i}^{-\alpha}+c\right)}\left(1-e^{-\lambda t_{0}^{-\alpha}}\right)^{n-n_{0}}
$$

Where, $C^{\star}$ is the normalizing constant. It is well established that the Bayes estimate of the parameter under squared error loss can be simply defined as the mean of the respective marginal posterior. The Bayes estimates of $\alpha$ and $\lambda$ can be obtained as

$$
\hat{\alpha}=C^{\star} \int_{0}^{\infty} \int_{0}^{\infty} \alpha^{n_{0}+b} \lambda^{n_{0}+d-1} \prod_{i=1}^{n_{0}} x_{i}^{-\alpha-1} e^{-\alpha a-\lambda\left(\sum_{i=1}^{n_{0}} x_{i}^{-\alpha}+c\right)}\left(1-e^{-\lambda t_{0}^{-\alpha}}\right)^{n-n_{0}} d \alpha d \lambda
$$

and

$$
\hat{\lambda}=C^{\star} \int_{0}^{\infty} \int_{0}^{\infty} \alpha^{n_{0}+b-1} \lambda^{n_{0}+d} \prod_{i=1}^{n_{0}} x_{i}^{-\alpha-1} e^{-\alpha a-\lambda\left(\sum_{i=1}^{n_{0}} x_{i}^{-\alpha}+c\right)}\left(1-e^{-\lambda t_{0}^{-\alpha}}\right)^{n-n_{0}} d \alpha d \lambda
$$

respectively. It can be seen that the above expressions $(8,9)$ cannot be obtained in nice closed form and one needs numerical approximation. Therefore, we proposed to use the Monte Carlo Markov chain (MCMC) methods namely Gibbs sampler [28, 30] and Metropolis Hastings algorithm [13, 3], to draw the random sample from the joint posterior so that sample based inference can be performed. For implementing the Gibbs algorithm, the full conditional posterior densities of $\alpha$ and $\beta$ are given by

$$
\begin{aligned}
& \pi_{1}(\alpha \mid \lambda, \underset{\sim}{x})=\alpha^{n_{0}+b-1} e^{-\alpha a-\lambda \sum_{i=1}^{n_{0}} x_{i}^{-\alpha}} \prod_{i=1}^{n_{0}} x_{i}^{-\alpha-1}\left(1-e^{-\lambda t_{0}^{-\alpha}}\right)^{n-n_{0}} \\
& \pi_{2}(\lambda \mid \alpha, \underset{\sim}{x})=\lambda^{n_{0}+d-1} e^{-\lambda\left(\sum_{i=1}^{n_{0}} x_{i}^{-\alpha}+c\right)}\left(1-e^{-\lambda t_{0}^{-\alpha}}\right)^{n-n_{0}}
\end{aligned}
$$

The Gibbs algorithm simulation process consist of the following steps:

Step 1. Start with $\mathrm{j}=1$ and initial value of $\lambda^{(0)}, \alpha^{(0)}$, then, at stage $\mathrm{j}$

Step 2. Using Metropolis-Hastings, generate $\alpha^{j} \sim \pi_{1}\left(\bullet \mid \lambda^{(j-1)}\right)$

Step 3. Using Metropolis-Hastings, generate $\lambda^{j} \sim \pi_{2}\left(\bullet \mid \alpha^{(j)}\right)$

Step 4. Repeat steps $2-3$ for all $\mathrm{j}=1,2, \ldots, \mathrm{M}$ and obtained $\left(\alpha_{1}, \lambda_{1}\right),\left(\alpha_{2}, \lambda_{2}\right), \ldots,\left(\alpha_{M}, \lambda_{M}\right)$. 
Step 5. The Bayes estimates of $\alpha$ and $\lambda$ under squared error loss, can be obtained as the mean of the simulated samples form their posteriors. Then, the formulae for obtaining the Bayes estimates of $\alpha$ and $\lambda$ are given by $\hat{\alpha}=\frac{1}{M} \sum_{i=1}^{M} \alpha_{i}$ and $\hat{\lambda}=\frac{1}{M} \sum_{i=1}^{M} \lambda_{i}$.

Once, we have the desired posterior sample from (7), the HPD credible intervals for $\alpha$ and $\lambda$ can be constructed by using algorithm given in [5]. Let $\left\{\Theta_{(i)} ; i=1,2, \ldots, M\right\}$ be the corresponding ordered MCMC sample of $\left\{\Theta_{i} ; i=\right.$ $1,2, \ldots, M\}$. Then, the $100(1-\psi) \%$ HPD intervals for $\Theta$ is $\left(\Theta_{\left(j^{*}\right)}, \Theta_{\left(j^{*}+[(1-\psi) M]\right)}\right)$, where $j^{*}$ is chosen so that

$$
\Theta_{\left(j^{*}+[(1-\psi) M]\right)}-\Theta_{\left(j^{*}\right)}=\min _{1 \leq j \leq M-[(1-\psi) M]}\left(\Theta_{(j+[(1-\psi) M])}-\Theta_{(j)}\right)
$$

Here, $[x]$ denotes the largest integer less than or equal to $x$. Then the HPD credible interval is that interval which has the shortest length.

\section{Comparison study}

This section consists the simulation study to check the performance of the discussed estimators on the basis of generated sample from IW distribution. The performance of the competitive estimators has been compared on the basis of their mean square errors (MSEs). For this purpose, we have generated a random sample of size $n(=20$ small, 30 moderate, 50 large $)$ from IW distribution for fixed values of $\alpha=1.5$ and $\lambda=2$. To view the effect of the hybrid censoring scheme on the performance of the estimators, we have taken different combinations of the censoring parameters $(R, T)$. The simulation results are summarised in Table 1 . To carry out this comparative study, we follow the following steps:

Step 1. Set $n, \alpha, \lambda, R$ and $T$.

Step 2. Generate $n$ standard uniform variates i.e. $U \sim \operatorname{Uniform}(0,1)$.

Step 3. Generate the sample of size $n$ from IW distribution by using the following formula $x=\left[-\frac{\log (u)}{\lambda}\right]^{-1 / \alpha}$.

Step 4. Apply Hybrid sampling procedure and obtain the hybrid Type-II censored sample of size $n_{0}, R \leq n_{0} \leq n$.

Step 5. Obtain the maximum likelihood estimates of $\alpha$ and $\lambda$ as $\hat{\alpha}$ and $\hat{\lambda}$

Step 6. Initialize the values of $\left\{\alpha^{(0)}, \beta^{(0)}\right\}$

Step 7. Take $a=b=c=d=0$ for non-informative prior (Bayes1), and $b / a=\alpha, d / c=\lambda$ for gamma prior (Bayes2).

Step 8. At stage $\mathbf{j}$, by using previous points, generate candidate points $\left\{\alpha_{c}^{(j)}, \beta_{c}^{(j)}\right\}$ from proposal densities $\left\{q_{1}\left(\alpha^{(j)} \mid \alpha^{(j-1)}\right)\right.$, $\left.q_{2}\left(\beta^{(j)} \mid \beta^{(j-1)}\right)\right\}$. Here, we take independent proposals for $\pi_{1}$ and $\pi_{2}$ as the asymptotic normal distributions of their MLEs.

Step 9. Generate $u \sim U(0,1)$ and set,

$\alpha^{(j)}= \begin{cases}\alpha_{c}^{(j)} & \text { if } u \leq \frac{\pi_{1}\left(\alpha_{c}^{(j)} \mid \beta^{(j-1)}, \underset{\sim}{x}\right) q_{1}\left(\alpha^{(j-1)} \mid \alpha_{c}^{(j)}\right)}{\pi_{1}\left(\alpha^{(j-1)} \mid \beta^{(j-1)}, \underset{\sim}{x}\right) q_{1}\left(\alpha_{c}^{(j)} \mid \alpha^{(j-1)}\right)} \\ \alpha^{(j-1)} & \text { otherwise }\end{cases}$

and then,

$\beta^{(j)}= \begin{cases}\beta_{c}^{(j)} & \text { if } u \leq \frac{\pi_{2}\left(\beta_{c}^{(j)} \mid \alpha^{(j)}, \underset{\sim}{x}\right) q_{2}\left(\beta^{(j-1)} \mid \beta_{c}^{(j)}\right)}{\pi_{2}\left(\beta^{(j-1)} \mid \alpha^{(j)}, \underset{\sim}{x}\right) q_{2}\left(\beta_{c}^{(j)} \mid \beta^{(j-1)}\right)} \\ \beta^{(j-1)} & \text { otherwise }\end{cases}$

Step 10. Repeat steps 8-9 for all $\mathrm{j}=1,2, \ldots, \mathrm{M}(=10000)$ and obtained $\left(\alpha_{1}, \lambda_{1}\right),\left(\alpha_{1}, \lambda_{1}\right), \ldots,\left(\alpha_{M}, \lambda_{M}\right)$.

Step 11. Under squared error loss, the Bayes estimate is the mean of the posterior distribution i.e. $\hat{\alpha}_{B} \approx \frac{1}{M-M_{0}}$ $\sum_{k=M_{0}+1}^{M} \alpha_{k}$ and $\hat{\lambda}_{B} \approx \frac{1}{M-M_{0}} \sum_{k=M_{0}+1}^{M} \lambda_{k}$, where, $M_{0}(=1000)$ is the burn-in-period of the Markov Chain. 
Step 12. Repeat steps 2-10, 1000 of times and obtain average maximum likelihood and Bayes estimates of the parameters and corresponding mean square errors by the following formulae, $\overline{\hat{\alpha}}=\sum \hat{\alpha} / 1000, M S E(\hat{\alpha})=$ $\sum(\hat{\alpha}-\alpha)^{2} / 1000$ and $\hat{\hat{\alpha}}_{B}=\sum \hat{\alpha}_{B} / 1000, \operatorname{MSE}\left(\hat{\alpha}_{B}\right)=\sum\left(\hat{\alpha}_{B}-\alpha\right)^{2} / 1000$.

From Table 1, the following concussions can be made,

- The MSEs of all the estimators (MLEs and Bayes) decreases as sample size $n$ increases i.e. all estimators become more efficient with increasing sample size.

- For fixed values of $n$, the MSEs of all the estimators decreases as censoring parameters $R$ and $T$ increases.

- In all the considered cases, the MSEs of the MLEs and Bayes estimators under non-informative and gamma priors can be ordered in the following way; $\boldsymbol{M S E}(\boldsymbol{m l e})>\boldsymbol{M S E}(\boldsymbol{B a y e s 1})>\boldsymbol{M S E}(\boldsymbol{B a y e s 2})$. Thus, the Bayes estimators perform well in the comparison of the MLEs.

Table 1: Average estimates and mean square errors (in brackets) of the estimators of $\alpha$ and $\lambda$ under different Type-II hybrid censoring schemes for fixed values of $\alpha=1.5, \lambda=2, a=2, b=3, c=2, d=4$

\begin{tabular}{|c|c|c|c|c|c|c|}
\hline $\mathrm{n}$ & $\mathrm{R}$ & $\mathrm{T}$ & Parameter & MLE & Bayes 1 & Bayes 2 \\
\hline \multirow{12}{*}{20} & \multirow{6}{*}{12} & \multirow{2}{*}{2} & $\alpha$ & $1.6121(0.131511)$ & $1.5264(0.102585)$ & $1.5006(0.066352)$ \\
\hline & & & $\lambda$ & $2.1962(0.566361)$ & $2.0579(0.365720)$ & $2.0043(0.149370)$ \\
\hline & & \multirow{2}{*}{3} & $\alpha$ & $1.5669(0.097831)$ & $1.4911(0.083916)$ & $2.047(0.0593110)$ \\
\hline & & & $\lambda$ & $2.182(0.4928310)$ & $1.4759(0.328080)$ & $2.0013(0.145390)$ \\
\hline & & \multirow{2}{*}{4} & $\alpha$ & $1.5558(0.087007)$ & $1.4885(0.077293)$ & $2.0397(0.057110)$ \\
\hline & & & $\lambda$ & $2.1695(0.431321)$ & $1.4759(0.299080)$ & $1.9994(0.144050)$ \\
\hline & \multirow{6}{*}{16} & \multirow{2}{*}{2} & $\alpha$ & $1.5792(0.094807)$ & $1.5145(0.079373)$ & $1.4971(0.057295)$ \\
\hline & & & $\lambda$ & $2.1741(0.439010)$ & $2.0468(0.309880)$ & $2.0036(0.148150)$ \\
\hline & & \multirow{2}{*}{3} & $\alpha$ & $1.5747(0.090734)$ & $1.5103(0.076180)$ & $1.4937(0.055195)$ \\
\hline & & & $\lambda$ & $2.1743(0.439180)$ & $2.0470(0.310050)$ & $2.0038(0.147900)$ \\
\hline & & \multirow{2}{*}{4} & $\alpha$ & $1.5651(0.083111)$ & $1.5021(0.071070)$ & $1.4876(0.052562)$ \\
\hline & & & $\lambda$ & $2.1708(0.423420)$ & $2.0440(0.300220)$ & $2.0027(0.146890)$ \\
\hline \multirow{12}{*}{30} & \multirow{6}{*}{18} & \multirow{2}{*}{2} & $\alpha$ & $1.5651(0.072468)$ & $1.5116(0.062890)$ & $1.501(0.0493320)$ \\
\hline & & & $\lambda$ & $2.1054(0.213250)$ & $2.0254(0.169080)$ & $2.0065(0.111241)$ \\
\hline & & \multirow{2}{*}{3} & $\alpha$ & $1.5328(0.057744)$ & $1.485(0.0538520)$ & $1.4792(0.043894)$ \\
\hline & & & $\lambda$ & $2.1021(0.207390)$ & $2.0224(0.165550)$ & $2.0052(0.109631)$ \\
\hline & & \multirow{2}{*}{4} & $\alpha$ & $1.5278(0.053274)$ & $1.4854(0.050393)$ & $1.4803(0.041893)$ \\
\hline & & & $\lambda$ & $2.0988(0.20299)$ & $2.0209(0.164400)$ & $2.0045(0.109991)$ \\
\hline & \multirow{6}{*}{24} & \multirow{2}{*}{2} & $\alpha$ & $1.5385(0.052523)$ & $1.4977(0.047765)$ & $1.4910(0.039616)$ \\
\hline & & & $\lambda$ & $2.0998(0.202680)$ & $2.0229(0.165190)$ & $2.0060(0.110840)$ \\
\hline & & \multirow{2}{*}{3} & $\alpha$ & $1.5377(0.051982)$ & $1.4970(0.047346)$ & $1.4904(0.039319)$ \\
\hline & & & $\lambda$ & $2.0999(0.202620)$ & $2.0230(0.165140)$ & $2.006(0.1108001)$ \\
\hline & & \multirow{2}{*}{4} & $\alpha$ & $1.5341(0.050689)$ & $1.4940(0.046696)$ & $1.4879(0.038975)$ \\
\hline & & & $\lambda$ & $2.0997(0.202510)$ & $2.0229(0.165210)$ & $2.006(0.1109010)$ \\
\hline \multirow{12}{*}{50} & \multirow{6}{*}{30} & \multirow{2}{*}{2} & $\alpha$ & $1.5564(0.049020)$ & $1.5249(0.044170)$ & $1.5190(0.037804)$ \\
\hline & & & $\lambda$ & $2.0766(0.128139)$ & $2.0309(0.111470)$ & $2.0212(0.087462)$ \\
\hline & & \multirow{2}{*}{3} & $\alpha$ & $1.5280(0.036777)$ & $1.4999(0.034744)$ & $1.4966(0.030500)$ \\
\hline & & & $\lambda$ & $2.0738(0.123241)$ & $2.0283(0.107639)$ & $2.0195(0.085089)$ \\
\hline & & \multirow{2}{*}{4} & $\alpha$ & $1.5247(0.033371)$ & $1.4999(0.031786)$ & $1.4970(0.028302)$ \\
\hline & & & $\lambda$ & $2.0715(0.120327)$ & $2.0268(0.105873)$ & $2.0185(0.084298)$ \\
\hline & \multirow{6}{*}{40} & \multirow{2}{*}{2} & $\alpha$ & $1.5311(0.033964)$ & $1.5071(0.031704)$ & $1.5036(0.028236)$ \\
\hline & & & $\lambda$ & $2.0725(0.120268)$ & $2.0281(0.106073)$ & $2.0197(0.084643)$ \\
\hline & & \multirow{2}{*}{3} & $\alpha$ & $1.5309(0.033892)$ & $1.5069(0.031650)$ & $1.5034(0.028192)$ \\
\hline & & & $\lambda$ & $2.0725(0.120258)$ & $2.0281(0.106066)$ & $2.0197(0.084638)$ \\
\hline & & \multirow{2}{*}{4} & $\alpha$ & $1.5279(0.032351)$ & $1.5041(0.030410)$ & $1.5009(0.027167)$ \\
\hline & & & $\lambda$ & $2.0717(0.119617)$ & $2.0274(0.105549)$ & $2.0191(0.084279)$ \\
\hline
\end{tabular}




\section{Bayesian prediction}

\subsection{One sample prediction}

In Type-II hybrid censoring scheme, some units out of $\mathrm{n}$ units put on test are not observed. In practice, the experimenter may be interested to know the life times of the removed surviving units on the basis of informative sample. Let $r<s \leq n$, from [1], the conditional distribution of sth order statistics given Type-II hybrid censored sample $x$, is given by

\section{For Case-I:}

$$
f\left(x_{s: n} \mid \underset{\sim}{x}, \alpha, \beta\right)=\frac{(n-R) !}{(n-s) !(s-R-1) !} \frac{\left(F\left(x_{s: n}\right)-F\left(x_{R: n}\right)\right)^{s-R-1}\left(1-F\left(x_{s: n}\right)\right)^{n-s} f\left(x_{s: n}\right)}{\left(1-F\left(x_{R: n}\right)\right)^{n-R}}
$$

Where, $x=\left\{x_{1}, x_{1}, \ldots, x_{R}\right\}, x_{s: n}>x_{R: n}$ and $R<s \leq n$.

Putting (1) and (2) in (12), we get

$$
\begin{aligned}
f\left(x_{s: n} \mid \underset{\sim}{x, \alpha, \beta)=}\right. & \frac{(n-R) !}{(n-s) !(s-R-1) !} \alpha \lambda x_{(s)}^{-\alpha-1} \sum_{i=0}^{s-R-1} \sum_{j=0}^{n-s}-1^{(i+j)}\left(\begin{array}{c}
s-R-1 \\
i
\end{array}\right)\left(\begin{array}{c}
n-s \\
j
\end{array}\right) \\
& \times \frac{\exp \left[-\lambda\left(i x_{(R)}^{-\alpha}+(s-R-i+j) x_{(s)}^{-\alpha}\right)\right]}{\left[1-\exp \left(-\lambda x_{(R)}^{-\alpha}\right)\right]^{n-R}}
\end{aligned}
$$

\section{For Case-II:}

$$
f\left(x_{s: n} \mid \underset{\sim}{x}, \alpha, \beta\right)=\sum_{k=R}^{s-1} \frac{(n-k) ! \operatorname{Pr}(K=k)}{(n-s) !(s-k-1) !} \frac{\left(F\left(x_{s: n}\right)-F(T)\right)^{s-k-1}\left(1-F\left(x_{s: n}\right)\right)^{n-s} f\left(x_{s: n}\right)}{\operatorname{Pr}(R \leq K \leq s-1)(1-F(T))^{n-k}}
$$

Where, $\underset{\sim}{x}=\left\{x_{1}, x_{1}, \ldots, x_{k}\right\}, x_{s: n}>T ; k<s \leq n$ and $\mathrm{K}$ is a random variable, represents the number of $x_{i}^{\prime} s$ that are at most $\tilde{\mathrm{T}}$. Then, we have

$$
P(K=k)=\left(\begin{array}{l}
n \\
k
\end{array}\right) F(t)^{k}[1-F(t)]^{n-k} ; \quad k=0,1, \ldots, n .
$$

By substituting (1) and (2) in (13), then equation (13) becomes

$$
\begin{aligned}
& f\left(x_{s: n} \mid \underset{\sim}{x, \alpha, \beta)}=\frac{\alpha \lambda x_{(s)}^{-\alpha-1}}{\operatorname{Pr}(R \leq K \leq s-1)} \sum_{k=R}^{s-1} \sum_{i=0}^{s-k-1} \sum_{j=0}^{n-s} \frac{(n-k) ! \operatorname{Pr}(K=k)}{(n-s) !(s-k-1) !}-1^{(i+j)}\right. \\
& \times\left(\begin{array}{c}
s-k-1 \\
i
\end{array}\right)\left(\begin{array}{c}
n-s \\
j
\end{array}\right) \frac{\exp \left[-\lambda\left(i T^{-\alpha}+(s-k-i+j) x_{(s)}^{-\alpha}\right)\right]}{\left[1-\exp \left(-\lambda T^{-\alpha}\right)\right]^{n-k}}
\end{aligned}
$$

The predictive posterior density of sth future observation under Type-II hybrid censoring scheme can be defined as

$$
f_{1}\left(y_{(s)} \mid \underset{\sim}{x}\right)=\int_{0}^{\infty} \int_{0}^{\infty} f\left(y_{(s)} \mid \alpha, \beta, \underset{\sim}{x}\right) \pi(\alpha, \beta \mid \underset{\sim}{x}) d \alpha d \beta
$$

From equations (13), (15) and (16), the predictive posterior of sth future sample is given by For Case I:

$$
\begin{aligned}
& f_{1}\left(x_{s: n} \mid \underset{\sim}{x}, \alpha, \beta\right)=\int_{0}^{\infty} \int_{0}^{\infty} \frac{C^{\star}(n-R) ! \alpha^{R-b} \lambda^{R-d} x_{(s)}^{-\alpha-1}}{(n-s) !(s-R-1) !} \prod_{i=1}^{R} x_{(i)}^{-\alpha-1} \sum_{i=0}^{s-R-1} \sum_{j=0}^{n-s}-1^{(i+j)}\left(\begin{array}{c}
n-s \\
j
\end{array}\right) \\
& \times\left(\begin{array}{c}
s-R-1 \\
i
\end{array}\right) \exp \left[-\lambda\left(i x_{(R)}^{-\alpha}+(s-R-i+j) x_{(s)}^{-\alpha}+\sum_{i=1}^{R} x_{(i)}^{-\alpha}+c\right)-\alpha a\right] d \alpha d \lambda
\end{aligned}
$$




\section{For Case II:}

$$
\begin{aligned}
f_{1}\left(x_{s: n} \mid \underset{\sim}{x}, \alpha, \beta\right) & =\int_{0}^{\infty} \int_{0}^{\infty} \frac{C^{\star}}{\operatorname{Pr}(R \leq K \leq s-1)} x_{(s)}^{-\alpha-1} \sum_{k=R}^{s-1} \sum_{i=0}^{s-k-1} \sum_{j=0}^{n-s} \alpha^{k-b} \lambda^{k-d} \prod_{i=1}^{k} x_{(i)}^{-\alpha-1} \\
& \times-1^{(i+j)} \frac{(n-k) !}{(n-s) !(s-k-1) !} \operatorname{Pr}(K=k)\left(\begin{array}{c}
s-k-1 \\
i
\end{array}\right)\left(\begin{array}{c}
n-s \\
j
\end{array}\right) \\
& \times \exp \left[-\lambda\left(i T^{-\alpha}+(s-k-i+j) x_{(s)}^{-\alpha}+\sum_{i=1}^{k} x_{(i)}^{-\alpha}+c\right)-\alpha a\right] d \alpha d \lambda
\end{aligned}
$$

Under square error loss, the Bayes estimates of the future samples are the mean of their posterior distributions and very difficult to obtain since the posteriors $(17,18)$ can not be tackled analytically. Therefore, we proposed to use the $\mathrm{M}-\mathrm{H}$ algorithm to draw the sample from the predictive posteriors $(17,18)$ and summarise the characteristics of the posteriors on the basis of that generated samples.

The two sided $100(1-\alpha) \%$ prediction intervals $\left(L_{s}, U_{s}\right)$ for $y_{(s)}$ by solving the following two equations:

$$
\begin{aligned}
& \operatorname{Pr}\left(y_{(s)}>U_{s} \mid \underset{\sim}{x}\right)=\frac{\alpha}{2} \\
& \operatorname{Pr}\left(y_{(s)}>L_{s} \mid \underset{\sim}{x}\right)=1-\frac{\alpha}{2}
\end{aligned}
$$

Confidence intervals can be obtained by using any suitable iterative procedure as above equations cannot be solved directly.

\subsection{Two sample prediction}

In some case, we are interested in the failure time of the sth ordered sample of size $\mathrm{N}$ from the same life time distribution and which is independent of informative sample i.e. $p\left(y_{(s)} \mid \alpha, \beta, \underset{\sim}{x}\right)=p\left(y_{(s)} \mid \alpha, \beta\right)$. This leads to the two sample prediction problems. The PDF of sth order statistics is given by

$$
p\left(y_{(s: N)} \mid \alpha, \beta\right)=\frac{N !}{(s-1) !(N-s) !}\left[F\left(y_{(s: N)}\right)\right]^{s-1}\left[R\left(y_{(s: N)}\right)\right]^{N-s} f\left(y_{(s: N)}\right)
$$

Putting (1) and (2) in (21), we get

$$
p\left(y_{(s: N)} \mid \alpha, \beta\right)=\frac{N !}{(s-1) !(N-s) !} \alpha \lambda y_{(s)}^{-\alpha-1} \sum_{j=0}^{N-s}-1^{j}\left(\begin{array}{c}
N-s \\
j
\end{array}\right) e^{-\lambda(s+j) y_{s}^{-\alpha}}
$$

Two sample predictive posterior density of sth future observation under Type-II hybrid censoring scheme is given by

$$
\begin{aligned}
p_{1}\left(y_{(s)} \mid \underset{\sim}{x}\right)= & C^{\star} s\left(\begin{array}{c}
N \\
s
\end{array}\right) \sum_{j=0}^{N-s}-1^{j}\left(\begin{array}{c}
N-s \\
j
\end{array}\right) \int_{0}^{\infty} \int_{0}^{\infty} \alpha^{n_{0}+b} \lambda^{n_{0}+d} y_{(s)}^{-\alpha-1} \prod_{i=1}^{n_{0}} x_{i}^{-\alpha-1}\left(1-e^{-\lambda t_{0}^{-\alpha}}\right)^{n-n_{0}} \\
& \exp \left[-\lambda\left((s+j) y_{s}^{-\alpha}+\sum_{i=1}^{n_{0}} x_{i}^{-\alpha}+c\right)-a \alpha\right] d \alpha d \beta
\end{aligned}
$$

The equation (23) cannot also be evaluated analytically. Therefore, to obtain the estimate of future sample, the $\mathrm{M}-\mathrm{H}$ algorithm is again used to draw the sample from equation (23). Then, we can estimate the two sample future observations under SELF as the mean of simulated sample drawn from (23). The survival function of future sample can be simply defined as

$$
S_{y}(T)=1-\int_{y=0}^{T} \int_{0}^{\infty} \int_{0}^{\infty} p\left(y_{(s)} \mid \alpha, \beta, \underset{\sim}{x}\right) \pi(\alpha, \beta \mid \underset{\sim}{x}) d \alpha d \beta d y
$$


We can also obtain the two sided $100(1-\alpha) \%$ prediction intervals $\left(L_{s}, U_{s}\right)$ for $y_{(s)}$ by solving the following two equations:

$$
\begin{aligned}
& P\left(y_{(s)}>U_{s} \mid \underset{\sim}{x}\right)=\frac{\alpha}{2} \\
& P\left(y_{(s)}>L_{s} \mid \underset{\sim}{x}\right)=1-\frac{\alpha}{2}
\end{aligned}
$$

Confidence intervals can be obtained by using any suitable iterative procedure as above equations cannot be solved directly. Alternatively, for obatining the predictive bounds of future samples, we can also use the sampling based approach developed by [5].

\section{$6 \quad$ Real data illustration}

In this section, to illustrate our discussed methodology, we have considered a data set representing the maximum flood levels (in millions of cubic feet per second) of the Susquehenna River at Harrisburg, Pennsylvenia over 20 four-year periods (1890-1969) as: $0.654,0.613,0.315,0.449,0.297,0.402,0.379,0.423,0.379,0.324,0.269,0.740$, $0.418,0.412,0.494,0.416,0.338,0.392,0.484,0.265$. [20] has discussed this data set to obtained the conditional confidence intervals for the parameters of IW distribution.

We have checked the suitability of the IW distribution to this real data set over more popular life-time models namely gamma, Weibull, flexible Weibull, and log-Normal. The model comparison criterion, Akaikes information criterion (AIC), Bayesian information criterion (BIC), and the measure of goodness-of-fit, K-S statistics are calculated from real data set by using the maximum likelihood estimates of the parameters of the above five models and summarised in Table 2. The Q-Q plots and fitted distribution functions of above four model for real data set are plotted in Figures 1. From Table 2 and Figure 1, one can easily conclude that the IW distribution gives better fit than the above four competitive distributions. For analysing this data set under Type-II hybrid censoring scheme, we have considered the two censoring schemes as:

Scheme 1: $\mathrm{R}=16, \mathrm{~T}=0.5$

Scheme 2: $\mathrm{R}=12, \mathrm{~T}=0.4$

For both censoring schemes, the maximum likelihood estimates and the Bayes estimates alongwith $95 \%$ confidence intervals (CI) and HPD credible intervals are obtained and presented in Table 3. For obtaining the Bayes estimates for real data set, non-informative prior is considered in the lack of prior information. The width of the CI and HPD intervals increases as $R$ and $T$ decreases although the width of the HPD intervals are smaller than that of CI.

For one and two sample predictive posteriors, we have considered the uniform proposals $U\left(X_{R}\right.$ or $\left.T, a_{1}\right)$ and $U\left(0, a_{2}\right)$ to the $\mathrm{MH}$ algorithm respectively. Where, $a_{1}$ and $a_{2}$ can be set by the experimenter at which a generated sequence reached to its stationary distributions with desirable acceptance rate. The sample based summary of one sample predictive densities of future failures for different values of $s$ has been presented in Tables 4 and 5 under scheme 1 and 2 respectively. Table 6 shows the characteristics of two sample predictive densities of future samples under scheme 2. From Tables 4-6, it is to be observed that the posterior standard error (PSE) of future ordered sample and corresponding width of the predictive bounds increase as $s$ increases.

Table 2: The model fitting summary of the above five distributions for flood data set

\begin{tabular}{cccccccc}
\hline Model & \multicolumn{2}{c}{ Estimates } & logL & AIC & BIC & K-S & Rank \\
\hline Weibull & 3.5265 & 0.4689 & 13.2664 & -22.5329 & -23.9308 & 0.4848 & 5 \\
Flexible W & 3.6456 & 0.8217 & 13.5886 & -23.1773 & -24.5752 & 0.2117 & 4 \\
Gamma & 26.9701 & 11.4124 & 15.1835 & -26.3669 & -27.7649 & 0.1611 & 3 \\
Log normal & -0.9020 & 0.2898 & 15.7177 & -27.4354 & -28.8334 & 0.1429 & 2 \\
Inverse W & 4.3140 & 0.0119 & 16.0974 & -28.1947 & -29.5927 & 0.1060 & 1 \\
\hline
\end{tabular}

\section{Conclusions}

In this paper, we have considered the estimation of the parameters of IW distribution under Type-II hybrid censored sample. The maximum likelihood and Bayes estimators of the parameters have been obtained and their performance 


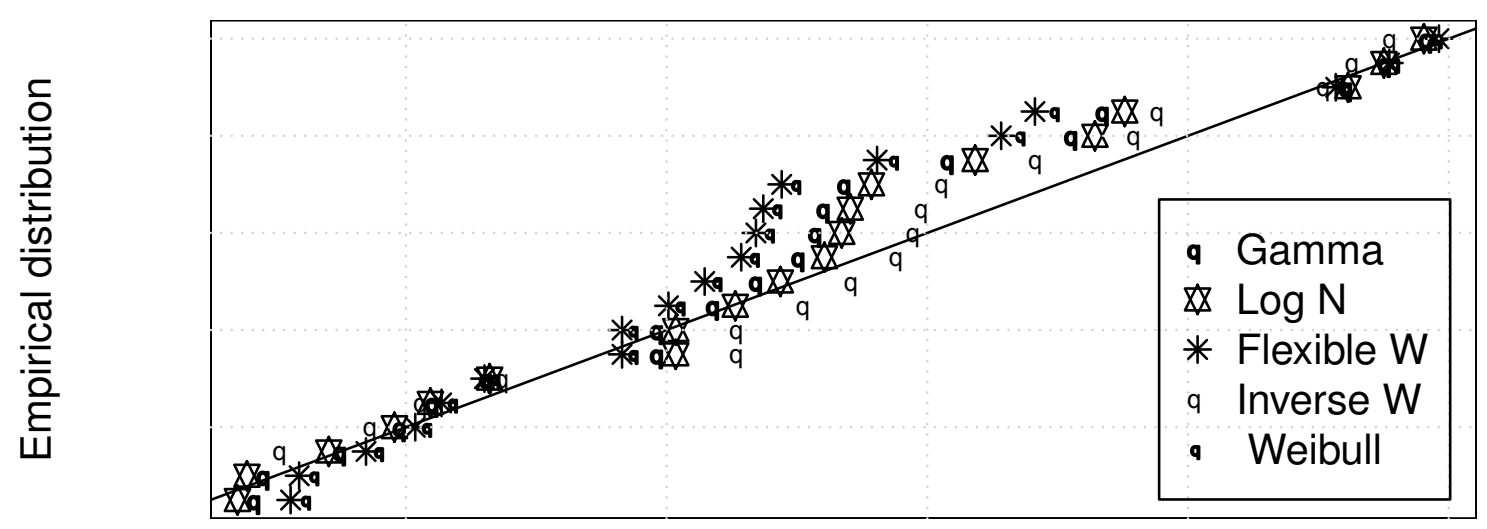

Fitted distribution

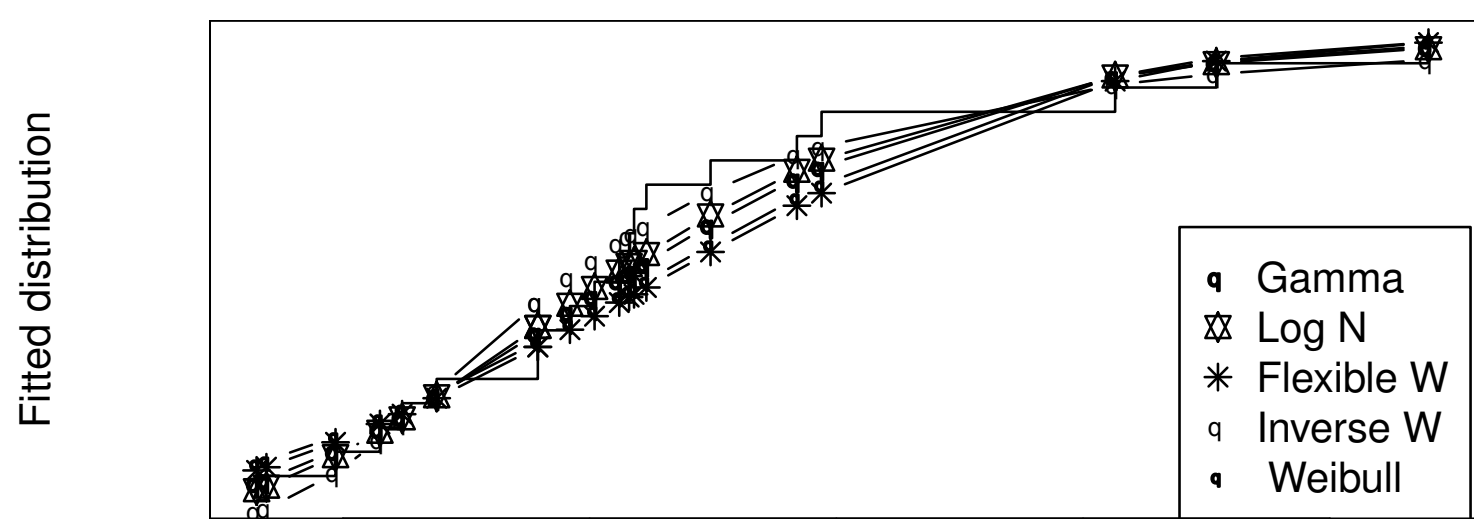

$\mathrm{X}$

Figure 1. The Q-Q plot (upper) and fitted distribution functions (lower) of various models from a set of flood data

Table 3: MlEs and Bayes estimates along with the corresponding 95\% CI and HPD intervals for flood data set

\begin{tabular}{cccccc}
\hline \multirow{2}{*}{ Scheme } & \multicolumn{3}{c}{ ML } & \multicolumn{2}{c}{ Bayes } \\
\cline { 3 - 6 } & & Estimate & CI & Estimate & HPD \\
\hline \multirow{2}{*}{ Complete } & $\alpha$ & 4.313991 & $2.654254,5.973734$ & 4.410332 & $3.436732,5.327457$ \\
& $\lambda$ & 0.011947 & $-0.011435,0.035331$ & 0.011902 & $0.002365,0.024636$ \\
\multirow{2}{*}{ Scheme 1 } & $\alpha$ & 4.418699 & $2.526157,6.311241$ & 4.454045 & $3.497427,5.395306$ \\
& $\lambda$ & 0.010546 & $-0.012982,0.034076$ & 0.011267 & $0.001956,0.023195$ \\
\multirow{2}{*}{ Scheme 2 } & $\alpha$ & 4.077173 & $2.070385,6.083961$ & 4.163735 & $3.289855,5.206142$ \\
& $\lambda$ & 0.015823 & $-0.021493,0.053140$ & 0.016031 & $0.003090,0.033417$ \\
\hline
\end{tabular}

Table 4: MCMC sample based summary of one sample predictive posteriors under scheme 1 for flood data set

\begin{tabular}{ccccc}
\hline \multirow{2}{*}{$\mathrm{s}$} & \multirow{2}{*}{ Mean } & \multirow{2}{*}{ PSE } & \multicolumn{2}{c}{$95 \%$ HPD Intervals } \\
\cline { 4 - 5 } & & & Lower & Upper \\
\hline 18 & 0.55884 & 0.052541 & 0.50001 & 0.66366 \\
19 & 0.61055 & 0.091015 & 0.50003 & 0.79986 \\
20 & 0.75151 & 0.193391 & 0.50144 & 1.15712 \\
\hline
\end{tabular}


Table 5: MCMC sample based summary of one sample predictive posteriors under scheme 2 for flood data set

\begin{tabular}{ccccc}
\hline \multirow{2}{*}{$\mathrm{s}$} & \multirow{2}{*}{ Mean } & \multirow{2}{*}{ PSE } & \multicolumn{2}{c}{$95 \%$ HPD Intervals } \\
\cline { 4 - 5 } & & & Lower & Upper \\
\hline 13 & 0.43163 & 0.015023 & 0.41602 & 0.46353 \\
14 & 0.45210 & 0.026073 & 0.41708 & 0.50292 \\
15 & 0.47552 & 0.035675 & 0.42108 & 0.54559 \\
16 & 0.50176 & 0.046063 & 0.42822 & 0.59420 \\
17 & 0.53910 & 0.059346 & 0.44061 & 0.66071 \\
18 & 0.58762 & 0.080822 & 0.45951 & 0.75596 \\
19 & 0.67484 & 0.129520 & 0.48367 & 0.95096 \\
20 & 0.86198 & 0.259530 & 0.51722 & 1.43230 \\
\hline
\end{tabular}

Table 6: MCMC sample based summary of the two sample predictive posteriors under scheme 2 for flood data set

\begin{tabular}{ccccc}
\hline \multirow{2}{*}{$\mathrm{s}$} & \multirow{2}{*}{ Mean } & \multirow{2}{*}{ PSE } & \multicolumn{2}{c}{$95 \%$ HPD Intervals } \\
\cline { 4 - 5 } & & & Lower & Upper \\
\hline 2 & 0.28711 & 0.025738 & 0.23694 & 0.33603 \\
4 & 0.31408 & 0.027078 & 0.26090 & 0.36633 \\
6 & 0.33806 & 0.028923 & 0.28483 & 0.40032 \\
8 & 0.36040 & 0.032128 & 0.29953 & 0.42621 \\
10 & 0.38588 & 0.034957 & 0.31679 & 0.45167 \\
12 & 0.41414 & 0.040832 & 0.33527 & 0.49688 \\
14 & 0.44992 & 0.047939 & 0.36253 & 0.54971 \\
16 & 0.49975 & 0.061374 & 0.38565 & 0.62105 \\
18 & 0.58461 & 0.091343 & 0.42558 & 0.77418 \\
20 & 0.86476 & 0.264890 & 0.48667 & 1.44140 \\
\hline
\end{tabular}

have been compared though Monte Carlo simulation. In all the considered cases, Bayes procedure provides the more efficient estimates of the model parameters with smaller confidence intervals width.

One and two sample predictive posteriors of future observations have been derived in the presence of Type-II hybrid censored sample. The predictive posterior becomes quit complicated as it contains many integrals and sums and can not be obtained in any standard closed form. The MCMC technique, Metropolis-Hastings algorithm, has been effectively utilized to draw the samples from predictive posteriors. Finally, the methodologies discussed in this paper can be recommended for their use where the IW distribution is applied.

\section{Acknowledgements}

The authors thank the referees and editor for their valuable comments and suggestions for improving the manuscript. The third author (Vikas Kumar Sharma) thanks UGC, New Delhi, India, for providing financial assistance.

\section{References}

[1] N. Balakrishnan and R.A. Shafay. One- and two-sample bayesian prediction intervals based on type-ii hybrid censored data. Communications in Statistics - Theory and Methods, 41:1511-1531, 2012.

[2] A. Banerjee and D. Kundu. Inference based on type-ii hybrid censored data from a weibull distribution. IEEE Transaction on Reliability, 57:369-378, 2008.

[3] S.P. Brooks. Markov chain monte carlo method and its application. Journal of the Royal Statistical Society: Series D, 47:69-100, 1998.

[4] R. Calabria and G. Pulcini. Bayes 2-sample prediction for the inverse weibull distribution. Communaication in Statistics- Theroy and Methods, 23, 1994.

[5] M.H. Chen and Q.M. Shao. Monte carlo estimation of bayesian credible and hpd intervals. Journal of Computational and Graphical Statistics, 6:66-92, 1998. 
[6] A. Childs, B. Chandrasekar, N. Balakrishnan, and D. Kundu. Exact likelihood inference based on type-i and type-ii hybrid censored sample from the exponential distribution. Annals of the Institute of Statistical Mathematics, 55:319-330, 2003.

[7] I.R. Dansmore. The bayesian predictive distribution in life testing models. Technometrics, 16:455-460, 1974.

[8] N. Draper and I. Guttman. Bayesina analysis of hybrid tests with exponential failure times. Annals of the Institute of Statistical Mathematics, 39:219-225, 1987.

[9] N. Ebrahmini. Prediction intervals for future failures in the exponential distribution under hybrid censoring. IEEE Transaction on Reliability, 41:127-132, 1992.

[10] B. Epstein. Truncated life test in the exponential case. Annals of Mathematical Statistics, 25:555-564, 1954.

[11] A. Ganguly, S. Mitra, D. Samanta, and D. Kundu. Exact inference for the two-parameter exponential distribution under type-ii hybrid censoring. Journal of Statistical Planning and Inference, 142:613-625, 2012.

[12] Puneet Kumar Gupta and Bhupendra Singh. Parameter estimation of lindley distribution with hybrid censored data. International Journal of System Assurance Engineering and Management, 1:1-8, 2012.

[13] W.K. Hastings. Monte carlo sampling methods using markov chains and their applications. Biometrika, 55:97-109, 1970.

[14] M.S. Khan, G.R. Pasha, and A.H. Pasha. Theoretical analysis of inverse weibull distribution. WSEAS Transictions on Mathematics, 7, 2008.

[15] Dal Ho Kim, Woo Dong Lee, and Sang Gil Kang. Non-infromative priors for inverse weibull distribution. Journal of Statistical Compuation and Simuation, iFirst:1-16, 2012.

[16] D. Kundu. On hybrid censored weibull distribution. Journal of Statistical Planning and Inference, 137:2127$2142,2007$.

[17] Debasis Kundua and Hatem Howlader. Bayesian inference and prediction of the inverse weibull distribution for type-ii censored data. Computational Statistics and Data Analysis, 54:1547-1558, 2010.

[18] A.O. Langlands, S.J. Pocock, G.R. Kerr, and S.M. Gore. Long-term survival of patients with breast cancer: a study of the curability of the disease. British Medical Journal, 2:1247-1251, 1997.

[19] J. F. Lawless. A prediction problem concerning samples from the exponential distribution, with application in life testing. Technometrics, 13:725-730, 1971.

[20] M. Maswadah. Conditional confidence interval estimation for the inverse weibull distribution based on censored generalized order statistics. Journal of Statistical Computation and Simulation, 73:887-898, 2010.

[21] MIL-STD-781C. Reliability design qualification and production acceptance test, exponential distribution. In U.S. Government printing office, Washington, DC, 1977.

[22] Farzana Noor and Muhammad Aslam. Bayesian inference of the inverse weibull mixture distribution using type-i censoring. Journal of Applied Statistics, 2013. doi:10.1080/02664763.2013.780157.

[23] Haniyeh Panahi and Saeid Asadi. Analysis of the type-ii hybrid censored burr type xii distribution under linex loss function. Applied Mathematical Sciences, 5:3929-3942, 2011.

[24] S. Park and N. Balakirshnan. A very flexible hybrid censoring scheme and its fisher information. Journal of Statistical Computation and Simulation, 82:41-50, 2012.

[25] R Core Team. R: A Language and Environment for Statistical Computing. R Foundation for Statistical Computing, Vienna, Austria, 2012. ISBN 3-900051-07-0, URL: http://www.R-project.org/.

[26] R.A. Shafay and N. Balakrishnan. One and two sample bayesian prediction intervals based on type-i hybrid censored data. Communication in Statistics - Simulation and Computation, 41:65-88, 2012.

[27] Sanjay Kumar Singh, Umesh Singh, and Dinesh Kumar. Bayesian estimation of parameters of inverse weibull distribution. Journal of Applied Statistics, 2013. DOI:10.1080/02664763.2013.789492. 
[28] A.F.M. Smith and G.O. Roberts. Bayesian computation via the gibbs sampler and related markov chain monte carlo methods. Journal of the Royal Statistical Society: Series B, 55:3-23, 1993.

[29] Khalaf S. Sultan and A. S. Al-Moisheer. Approximate bayes estimation of the parameters and reliability function of a mixture of two inverse weibull distributions under type-2 censoring. Journal of Statistical Computation and Simulation, 2012. doi:10.1080/00949655.2012.673614.

[30] S.K. Upadhyay, N. Vasishta, and A.F.M. Smith. Bayes inference in life testing and reliability via markov chain monte carlo simulation. Sankhya A, 63:15-40, 2001. 